Reprod. Nutr. Dévelop., 1981, 21 (2), 279-287.

\title{
Etude des flux de leucine : total, catabolisme oxydatif, synthèse et catabolisme protéique, au cours du développement du rat
}

\author{
par Christiane OBLED, Monique ALRIC, M. ARNAL, G. FAUCONNEAU \\ Laboratoire d'Ełude du Méfabolisme Azoté \\ I.N.R.A., Theix, 63110 Beaumont, France.
}

Summary. Leucine flux : total flux, oxidative catabolism, protein synthesis and breakdown during development in the rat.

Total leucine flux (flux into or out of the free leucine pool) and its different components - oxidative catabolism, protein synthesis and protein degradation - were studied in male rats weighing 160 and $300 \mathrm{~g}$ and infused intravenously for $6 \mathrm{~h}$ with $\mathrm{L}-\left({ }^{14} \mathrm{C}\right)$ leucine. Plasma L- $\left({ }^{14} \mathrm{C}\right)$ leucine specific activity was measured after 4 and $6 \mathrm{~h}$ of infusion. The excretion rate of ${ }^{14} \mathrm{CO}_{2}$ was estimated during infusion. Total leucine flux and oxidative catabolism flux were calculated from the specific radioactivity in the plasma at plateau value. Rates of protein synthesis were calculated from the total flux after subtracting the proportion oxidized. Protein breakdown flux was estimated by the difference between total flux and dietary intake flux.

Total leucine flux ( $\mu$ moles $/ \mathrm{h} / 100 \mathrm{~g}$ body weight) was not significantly lower at 300 than at $160 \mathrm{~g}$. Oxidation accounted for about $10 \mathrm{p} .100$ of the leucine flux; this proportion was significantly larger at 300 than at $160 \mathrm{~g}$.

A comparison of protein synthesis flux between the two groups of tats, for the same value of total flux, showed a decrease of leucine utilization for protein synthesis in the older animals.

The quantity of leucine provided by protein breakdown was comparatively more important at 300 than at $160 \mathrm{~g}$. Protein deposition fell by about 50 p. 100 between these two weights.

\section{Introduction.}

La quantité de protéines fixée par l'animal au cours de la croissance résulte de l'importance relative de la quantité d'acides aminés engagés dans différentes voies métaboliques, synthèse protéique, catabolisme oxydatif et protéique.

Le flux total des acides aminés, c'est-à-dire la quantité totale d'un acide aminé qui entre ou sort du pool libre par unité de temps (Waterlow et Stephen, 1967), peut être facilement mesuré par la méthode de perfusion continue. Le flux total ainsi que le flux de synthèse (quantité tolale de l'acide aminé incorporé dans les protéines par unité de temps) ont été assez bien étudiés chez différentes espèces comme le rat (Waterlow et Stephen, 1967 ; Garlick, Millward et James, 1973 ; Lo et Millward, 1977; Waterlow, 
Garlick ef Millward, 1978 ; Albertse, Garlick et Pain, 1979 ; Reeds et Lobley, 1980), le lapin (Nicholas, Lobley et Harris, 1977 ; Reeds et Lobley, 1980), le porc (Edmunds ef Buttery, 1978 ; Reeds ef al., 1978 ; Garlick, Burk et Swick, 1976), l'homme (James et al., 1976, Golden et Waterlow, 1977 ; Garlick, Burk et Swick, 1976). Le flux de catabolisme oxydatif (quantité d'acide aminé oxydé par unité de temps) a été moins étudié (James et al., 1976 ; Garlick et al., 1978 ; Reeds et al., 1978 ; Albertse, Garlick et Pain, 1979), car de nombreux auteurs expriment seulement la quantité de ${ }^{14} \mathrm{CO}_{2}$ excrété en pourcentage de la dose injectée. Il existe peu de mesures au cours du développement (Waterlow et Stephen, 1967 ; Waterlow, Garlick et Millward, 1978). Le but du présent travail est d'étudier les différents flux de leucine chez le rat à deux stades de son développement.

\section{Matériel et méthodes.}

Deux lots de rats mâles, de souche Sprague Dawley, sont élevés en cage individuelle, dans des locaux éclairés de $7 \mathrm{~h}$ à $19 \mathrm{~h}$, maintenus à $22^{\circ} \mathrm{C}$ et de degré hygrométrique contrôlé. Ils consomment ad libitum un régime semi-synthétique à base de farine de poisson contenant 11,7 p. 100 de matières azotées $(N \times 6,25)$ par rapport à la matière sèche (Grizard ef al., 1975). A un poids moyen de $80 \mathrm{~g}$ environ pour les rats $\mathrm{du}$ lot 1 et $200 \mathrm{~g}$ pour les rats du lot 2, les animaux reçoivent les aliments en 6 repas égaux par jour, distribués à $11,15,19,23,3$ et $7 \mathrm{~h}$.

Une semaine environ avant la perfusion, deux canules sont implantées sur les animaux sous anesthésie, l'une dans la jugulaire droite, l'autre dans la carotide droite, selon une méthode voisine de celles décrites par Weeks (1972) et Jarrige, Boucher et Leinot (1978). L'opération n'a aucune influence sur la croissance ef la consommation des animaux du lot 1. Par contre, les animaux plus âgés récupèrent plus difficilement (tabl. 1).

Le jour de la perfusion, le rat est placé dans une chambre d'un litre de volume balayée par un courant d'air. Les canules sortant au niveau de la nuque du rat sont reliées à l'extérieur de la cage par l'intermédiaire de cathéters en polyéthylène passant à l'intérieur d'un ressort fixé lui-même sur le rat par l'intermédiaire d'un harnais. Le port du harnais empêche le rat de se retourner dans la cage ef d'endommager les catheters. Les animaux sont habitués pendant environ 10 jours au port du harnais d'une part et d'autre part à séjourner dans la chambre de mesure.

Une solution de leucine $1{ }^{14} \mathrm{C}(54 \mathrm{mCi} / \mathrm{mM})$ est perfusée dans la veine jugulaire à une vitesse d'environ $3-4 \mu \mathrm{Ci} / \mathrm{h}$ pour les animaux de $160 \mathrm{~g}$ et $6-7 \mu \mathrm{Ci} / \mathrm{h}$ pour les rats de $300 \mathrm{~g}$. La perfusion a lieu de $9 \mathrm{~h}$ à $15 \mathrm{~h}$. Deux prélèvements de sang dans la carotide sont réalisés respectivement après $4 \mathrm{~h}$ et $6 \mathrm{~h}$ de perfusion. Pendant toute la durée de la perfusion, le gaz carbonique expiré par l'animal est mesuré par un analyseur à infrarouge et recueilli ensuite dans une solution de soude $0,8 \mathrm{~N}$. La radioactivité de cette solution est déterminée sur des fractions aliquotes prélevées au cours de la perfusion.

A la fin de la perfusion, le rat est rapidement abattu par égorgement sans anesthésie. Le sang prélevé sur héparine est rapidement centrifugé $(5 \mathrm{~min}-3000 \mathrm{~g})$. Le plasma et la carcasse éviscérée et sans peau, après broyage dans l'azote liquide, sont placés dans 8 volumes d'acide trichloracétique 10 p. 100 (W/V) (ATC) froid, de façon 
TABLEAU 1

Croissance, consommation et efficacité alimentaire

Lot

1

2

\begin{tabular}{|c|c|c|c|c|}
\hline Nombre d'animaux $\ldots \ldots \ldots \ldots$ & 10 & & 7 & \\
\hline Poids moyen initial (g) & 100 & (4) & 237 & (7) \\
\hline $\begin{array}{l}\text { Poids moyen au moment de la perfusion } \\
\text { (g) } \ldots \ldots \ldots \ldots \ldots \ldots \ldots \ldots \ldots \ldots \ldots \ldots \ldots\end{array}$ & 162 & (6) & 300 & (3) \\
\hline 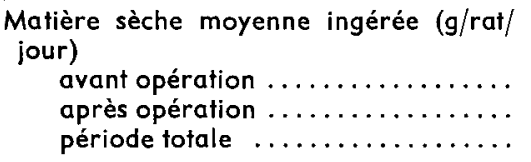 & $\begin{array}{l}12,8 \\
14,5 \\
13,6\end{array}$ & $\begin{array}{l}(0,9)^{a c} \\
(1,1)^{b} \\
(0,7)^{c b}\end{array}$ & $\begin{array}{l}19,1 \\
16,5 \\
17,4\end{array}$ & $\begin{array}{l}(0,8)^{d f} \\
(1,7)^{e} \\
(1,6)^{e f}\end{array}$ \\
\hline 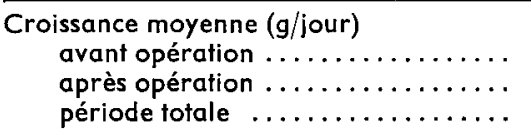 & $\begin{array}{l}4,5 \\
5,3 \\
4,8\end{array}$ & $\begin{array}{l}(0,3)^{a} \\
(1,2)^{a} \\
(0,7)^{a}\end{array}$ & $\begin{array}{l}5,6 \\
2,5 \\
4,1\end{array}$ & $\begin{array}{l}(0,9)^{a} \\
(1,5)^{b c} \\
(1,0)^{a c}\end{array}$ \\
\hline 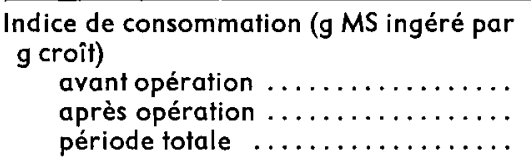 & $\begin{array}{l}2,9 \\
2,9 \\
2,9\end{array}$ & $\begin{array}{l}(0,2)^{a} \\
(0,6)^{a} \\
(0,3)^{a}\end{array}$ & $\begin{array}{l}3,5 \\
8,7 \\
4,4\end{array}$ & $\begin{array}{l}(0,6)^{a} \\
(5,0)^{b c} \\
(0,8)^{a c}\end{array}$ \\
\hline
\end{tabular}
5 p. 100.

(Ecart type) - Les chiffres affectés d'une lettre différente sont significativement différents au seuil

à extraire les acides aminés libres (Obled, Arnal ef Fauconneau, 1975). L'activité spécifique de la leucine libre esł déterminée après séparation sur un analyseur automatique suivi d'un spectromètre à cellule d'anthracène (Packard Flow Defector).

Au cours de la perfusion d'un acide aminé marqué, l'évolution de l'activité spécifique dans le plasma et les différents tissus peut être assimilée à une fonction du type $S_{p}=S_{p} \max \left(1-e^{-\lambda . p t}\right)$ (Waterlow et Stephen, 1967) $\left(S_{p}=\right.$ activité spécifique de l'acide aminé dans le plasma, $S p$ max $=$ activité spécifique maximum, $\lambda p=$ constante de vitesse, $t=$ temps). Le flux total qui représente la quantité de leucine entrant ou quittant le pool libre plasmatique par unité de temps, est calculé à partir de l'équation suivante $: \mathrm{I}=\mathrm{S}_{\mathrm{p}} \max \times \mathrm{F}$ (Waterlow et Stephen, 1967 ; Waterlow, Garlick et Millward, 1978) $(\mathrm{I}=$ vitesse de perfusion de l'isotope $(\mu \mathrm{Ci} / \mathrm{h}) ; \mathrm{F}=$ flux total de l'acide aminé ( $\mu$ Mole/h).

L'activité spécifique du ${ }^{14} \mathrm{CO}_{2}$ expiré atteint une valeur maximale au cours de la perfusion et le flux de catabolisme oxydatif $C(\mu \mathrm{mole} / \mathrm{h})$ est déterminé à partir de la vitesse moyenne d'excrétion de l'isotope au plateau $E: E=S p \max \times C$ (O'Keefe. Sender et James, 1974 ; James ef al., 1976).

Le flux de synthèse $S$ et de dégradation des protéines $B$ sont calculés par différence connaissant le flux alimentaire $A$ à partir de l'égalité suivante $: F=C+S=A+B$ (O'Keefe, Sender et James, 1974 ; James et al., 1976). 
Résultats (łabl. 2).

Le flux total de leucine ramené à $100 \mathrm{~g}$ de poids vif ne diminue pas significativement entre 160 et $300 \mathrm{~g}$.

TABLEAU 2

Flux de leucine chez le rat ò deux stades de son développement

\begin{tabular}{|c|c|c|c|}
\hline \multicolumn{2}{|l|}{ Lot } & $160 \mathrm{~g}$ & $300 \mathrm{~g}$ \\
\hline \multicolumn{4}{|c|}{ ( $\mu$ mole leucine $/ \mathrm{h} .100 \mathrm{~g}$ ) } \\
\hline $\begin{array}{l}\text { Flux total : } \\
\text { Flux catabolisme oxydatif : } \\
\text { Flux synthèse protéique : } \\
\text { Flux alimentaire : } \\
\text { Flux catabolisme protéique : }\end{array}$ & 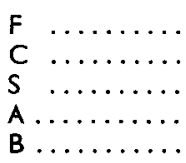 & $\begin{aligned} 56,8 a & \pm 16,6 \\
5,3 a & \pm 1,6 \\
49,9 a & \pm 15,5 \\
23,6^{a} & \pm 1,8 \\
31,7 a & \pm 16,1\end{aligned}$ & $\begin{aligned} 53,7 a & \pm 11,5 \\
6,8 a & \pm 1,0 \\
46,7^{a} & \pm 10,5 \\
14,7^{b} & \pm 1,6 \\
39,0 a & \pm 10,8\end{aligned}$ \\
\hline
\end{tabular}

(g protéine/jour. $100 \mathrm{~g}$ )

\begin{tabular}{|c|c|c|c|}
\hline 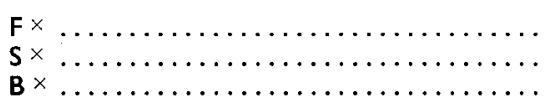 & $\begin{array}{l}2,67 a \pm 0,80 \\
2,41 a \pm 0,73 \\
1,52^{a} \pm 0,78\end{array}$ & $\begin{array}{l}2,20 a \pm \\
1,91 a \pm \\
1,64 a \pm\end{array}$ & $\begin{array}{l}0,48 \\
0,43 \\
0,48\end{array}$ \\
\hline $\begin{array}{l}S-B \text { (g prot/jour) } \ldots \ldots \ldots \ldots \ldots \ldots \ldots \\
S-B / \text { croît journalier }(\text { p. 100) } \ldots \ldots \ldots \ldots \ldots\end{array}$ & $1,43 a_{27}^{a} 0,26$ & $0,79 b \pm$ & 0,23 \\
\hline
\end{tabular}

constantes de vitesse (p. 100/jour)

Synthèse : $\quad \mathrm{ks}^{+} \ldots \ldots \ldots \ldots \ldots \ldots \ldots \ldots$

Catabolisme $: \mathrm{kd}^{+} \ldots \ldots \ldots \ldots \ldots \ldots \ldots$

10

9

\pm Ecart type.

${ }^{\times}$Teneur en leucine des protéines corporelles $: 6,6 \mathrm{~g} / 16 \mathrm{gN}$ à $160 \mathrm{~g}, 7,2 \mathrm{~g} / 16 \mathrm{gN}$ à $300 \mathrm{~g}$.

+ Quantité de protéines dans l'organisme : $28,0 \mathrm{~g}$ à $160 \mathrm{~g}$ (Grizard et al., 1975) et $55,5 \mathrm{~g}$ à $300 \mathrm{~g}$ (Rérat ef al., 1964).

Les chiffres affectés d'une lettre différente sont significativement différents au seuil 5 p. 100.

La radioactivité excrétée dans le ${ }^{14} \mathrm{CO}_{2}$ augmente rapidement après le début de la perfusion de leucine $1{ }^{14} \mathrm{C}$ jusqu'à une valeur maximale atteinte après environ $2 \mathrm{~h} 30$ chez les animaux du lot 1 et $4 \mathrm{~h}$ chez les animaux du lot 2 (Alric, 1979). L'ajustement des données expérimentales à une fonction du type $s c=s c \max \left(1-\mathrm{e}^{-\lambda c t}\right)$ donne des valeurs moyennes de $\lambda c$ égales à $39 \mathrm{j}^{-1}$ pour les rats de $160 \mathrm{~g}$ et $24 \mathrm{j}^{-1}$ pour les rats de $300 \mathrm{~g}$. Au plateau, la vitesse d'excrétion du ${ }^{14} \mathrm{CO}_{2}$ exprimée en $\mathrm{p}$. 100 de la dose perfusée par unité de temps est significativement plus élevée pour les animaux de $300 \mathrm{~g}$ $(11,1 \mathrm{p} .100 / \mathrm{h})$ que pour les animaux de $160 \mathrm{~g}(7,7 \mathrm{p} .100 / \mathrm{h})$. Le flux de catabolisme oxydatif ( $\mu$ moles $/ \mathrm{h} .100 \mathrm{~g}$ ) n'augmente pas significativement entre 160 et $300 \mathrm{~g}$.

Le flux de synthèse protéique, obtenu par différence entre le flux total ef le flux de catabolisme oxydatif, ne diminue pas significativement entre les deux stades expérimentaux.

Le flux alimentaire diminue d'environ 50 p. 100 entre 160 ef 300 g. Pour une 
même quantité ingérée, l'alimentation par repas modifie la vitesse de croissance ef améliore l'indice de consommation comparativement à l'alimentation ad libitum (Alric, 1979). Malgré un ralentissement de la vitesse de croissance due à l'opération pour les animaux de $300 \mathrm{~g}$, nous obtenons le même résultat que dans des études précédentes (Obled, Arnal ef Fauconneau, 1975).

Le flux de catabolisme protéique ne varie pas significativement entre les deux âges expérimentaux.

La quantité de protéines fixées par l'animal, appréciée par la différence entre le flux de synthèse et le flux de catabolisme protéique, diminue d'environ 50 p. 100 entre 160 et $300 \mathrm{~g}$.

La constante de vitesse de synthèse protéique (p. 100/jour) est plus faible à $300 \mathrm{~g}$ qu'à $160 \mathrm{~g}$. La constante de vitesse de dégradation ne diminue pas entre les deux stades expérimentaux.

\section{Discussion.}

- Choix du pool de leucine libre.

Le calcul des différents flux au niveau de l'organisme nécessite la détermination de l'activité spécifique de l'acide aminé libre total de l'organisme. En fait, la plupart des auteurs utilisent l'activité spécifique du précurseur libre plasmatique, ce qui entraîne une sous-estimation des différents flux, étant donné que l'activité spécifique des acides aminés libres dans les tissus est toujours inférieure à celle du plasma. De façon à apprécier l'importance de la sous-estimation, nous avons calculé le flux total et le flux de catabolisme oxydatif en considérant l'activité spécifique de la leucine libre dans le corps eviscéré et sans peau. Cette activité spécifique tissulaire représente 43 p. 100 de l'activité spécifique plasmatique à $160 \mathrm{~g}$ et $56 \mathrm{p} .100 \mathrm{d̀} 300 \mathrm{~g}$ ce qui entraîne des valeurs du flux total et du flux de catabolisme oxydatif deux fois plus élevées.

La quantité de protéines fixées par l'animal, estimée par la différence entre les flux de synthèse et de catabolisme protéique, représente 30 p. 100 du croît journalier lorsqu'elle est calculée à partir du pool plasmatique et 15 p. 100 à partir du pool tissulaire (assimilé au pool de leucine libre dans le corps éviscéré et sans peau). La quantité de protéines fixées déterminée par des méthodes de bilans azotés représente environ 20 p. 100 du croît journalier (Vermorel, communication personnelle).

Il semblerait que l'activiłé spécifique du véritable pool précurseur soit intermédiaire entre l'activité spécifique du pool extracellulaire, appréciée par l'activité spécifique plasmatique, et du pool intracellulaire, appréciée par l'activité spécifique intratissulaire.

Peu d'auteurs détermineni le flux de catabolisme oxydatif et assimilent le flux de synthèse protéique au flux total en supposant que les deux erreurs introduites, d'une part en négligeant le flux de catabolisme oxydatif, d'autre part par la sous-estimation du flux déterminé à partir de l'activité spécifique du précurseur dans le plasma, se compensent. Il apparaît que cette hypothèse n'est pas vérifiée dans le cas de la leucine, le flux de catabolisme oxydatif ne représentant qu'environ 10 p. $100 \mathrm{du}$ flux total, alors que la sous-estimation du flux total est d'environ 50 p. 100. 
- Influence de l'âge sur les différents flux.

De façon générale, nos valeurs du flux total et du flux de synthèse protéique sont plus faibles que celles déterminées par d'autres auteurs avec la tyrosine et la lysine. Elles sont assez proches de celles citées par les auteurs ayant utilisé la leucine (fig. 1).
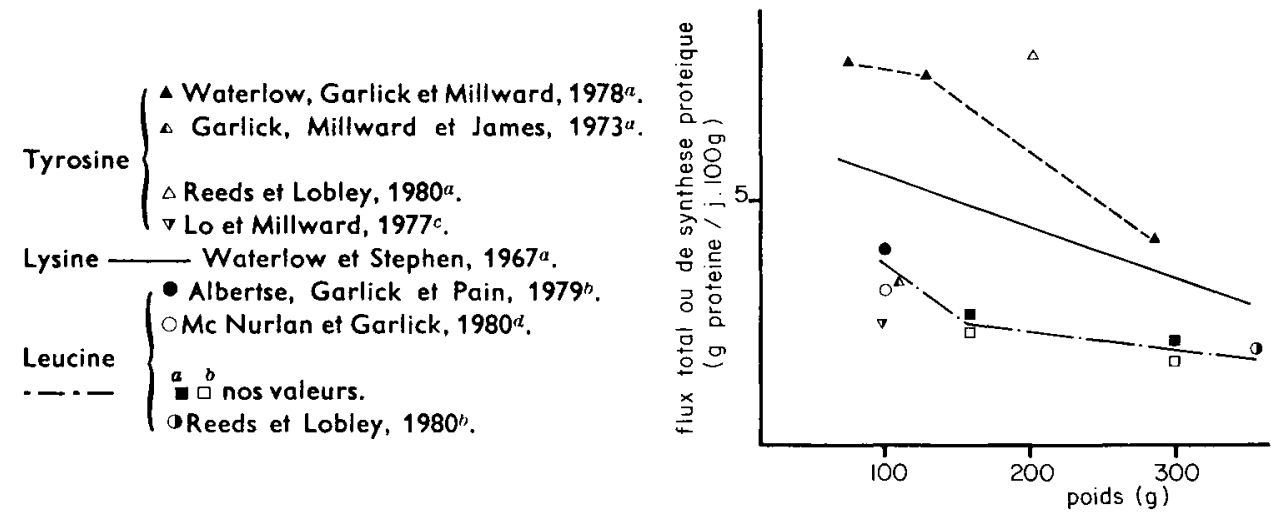

FIG. 1. - Flux tofal ef flux de synthèse protéique chez le rat ou cours du développement, estimés par perfusion continue de divers acides marqués. $a$ : flux total $;^{i}:$ flux de synthèse protéique estimé par différence entre le flux total et le flux de catabolisme oxydatif ; ${ }^{c}$ : flux de synthèse protéique estimé à partir de la détermination de la constante de synthèse ks sur l'animal entier; ${ }^{d}:$ comme $c$ avec une dose élevée de l'acide aminé marqué.

Le flux de catabolisme oxydatif de la leucine est voisin de celui déterminé par Neale et Waterlow (1973) chez le rat de $100 \mathrm{~g}(6,4 \mu$ moles/jour. $100 \mathrm{~g})$ et plus faible que celui déterminé par Albertse, Garlick et Pain (1979) chez le rat de $100 \mathrm{~g}(13,3 \mu$ moles $/ \mathrm{h} .100 \mathrm{~g})$ Les valeurs plus faibles que nous trouvons peuvent provenir du fait que les animaux sont nourris par repas, perfusés pendant le jour (Obled, Arnal et Fauconneau, 1975) et reçoivent un régime à faible teneur en protéines.

Les différents flux varient peu au cours du développement contrairement aux résultats cités par Millward (Waterlow, Garlick et Millward, 1978) avec la tyrosine et Waterlow et Stephen (1967) avec la lysine entre les deux stades expérimentaux. Le flux total exprimé par $\mathrm{kg}^{0,75}$ est de $16,9 \mathrm{~g}$ protéines/jour à $160 \mathrm{~g}$ et 16,3 à $300 \mathrm{~g}$.

Le flux de synthèse varie de 15,6 et $14,1 \mathrm{~g}$ protéines/jour $\mathrm{kg}^{0,75}$. Ces valeurs sont voisines de celles citées par Reeds ef Lobley (1980), $16,7 \mathrm{~g}$ protéines $/ \mathrm{g} \mathrm{kg}{ }^{0}, 75$ chez le rat de $350 \mathrm{~g}$, par contre elles sont plus faibles que celles obtenues par Millward (Waterlow, Garlick et Millward, 1978). Il semble qu'il y aurait une meilleure relation entre le flux total ou le flux de synthèse ef le poids vif porté à la puissance 0,56 (12,5 et 12,9 pour le flux total et 11,0 et $11,2 \mathrm{~g}$ protéine/jour. $\mathrm{kg}^{0,56}$ pour le flux de synthèse aux 2 stades expérimentaux). Il faudrait toutefois plus de stades expérimentaux pour vérifier cette relation.

A un poids donné, nous observons une variabilité individuelle très importante, 
les coefificients de variation des différents paramètres mesurés allant de 15 à 30 p. 100 . Les corrélations entre les différents flux ef le flux total pour chaque animal à l'intérieur d'un même lot font apparaître que la proportion de leucine engagée dans chaque voie métabolique est relativement constante (tabl. 3). Les droites obtenues dans les deux

\section{TABLEAU 3}

Correlations des différents flux en fonction du flux fofal de leucine chez le rat à deux stades de son développement

\begin{tabular}{|c|c|c|}
\hline Lot & $162 \mathrm{~g}$ & $300 \mathrm{~g}$ \\
\hline 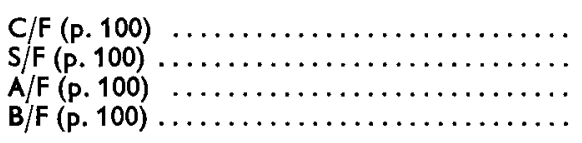 & $\begin{array}{r}9,7 \\
90,3 \\
44,8 \\
52,7\end{array}$ & $\begin{array}{l}12,9 \\
86,9 \\
28,2 \\
71,8\end{array}$ \\
\hline \multicolumn{3}{|c|}{ Différentes régressions + } \\
\hline 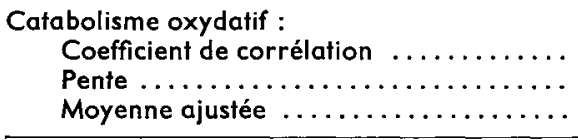 & $\begin{array}{l}0,82 \\
0,086 * a \\
5,2 a \pm 0,2\end{array}$ & $\begin{array}{l}0,90 \\
0,080 * a \\
6,9^{b} \pm 0,2\end{array}$ \\
\hline 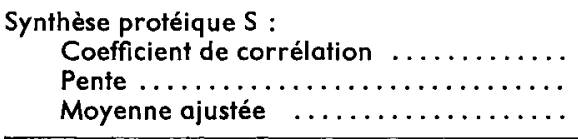 & $\begin{array}{l}0,99 \\
0,91^{* a} \\
49,3^{a} \pm 0,2\end{array}$ & $\begin{array}{l}0,99 \\
0,91^{* a} \\
47,5^{b} \pm 0,2\end{array}$ \\
\hline 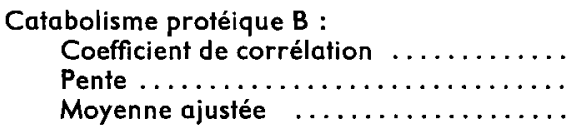 & $\begin{array}{l}0,96 \\
0,87 * a \\
30,5^{a} \pm 0,9\end{array}$ & $\begin{array}{l}0,98 \\
0,93 * a \\
40,7^{b} \pm 1,2\end{array}$ \\
\hline
\end{tabular}

* Valeur significative au seuil 5 p. 100.

$+\mathrm{C}, \mathrm{S}, \mathrm{B}=\mathrm{dF}+\mathrm{f}$.

$a, b$ Les chiffres affectés d'une lettre différente sont sifnificativement différentes au seuil 5 p. 100.

lots sont parallèles; la comparaison des moyennes ajustées (Snedecor et Cochran, 1971) fait apparaître que comparativement les animaux plus âgés perdent plus de leucine par oxydation. De ce fait la synthèse protéique diminue. Comme l'apport alimentaire contribue pour une moindre part au flux total, il y a plus d'acides aminés provenant du catabolisme des protéines. La quantité de protéines fixées diminue en fonction de l'âge, d'une part parce que la constante de vitesse de synthèse diminue alors que la constante de vitesse de dégradation des protéines ne varie pas, d'autre part parce que la quantité totale de protéines synthétisées par l'animal augmente moins que la quantité dégradée. L'augmentation du catabolisme oxydatif de la leucine peut provenir d'une part du fait que les animaux ont consommé le même régime alors que les animaux plus âgés ont besoin d'une proportion moins importante d'acides aminés indispensables (Smith, 1980) et d'autre part du fait que les rats plus âgés fixent moins de protéines. 


\section{Conclusion.}

Entre 100 et $300 \mathrm{~g}$, il semble que le flux total diminue de façon plus ou moins importante selon l'acide aminé utilisé (fig. 1), en particulier le flux total de leucine varie peu entre 160 et $300 \mathrm{~g}$. La répartition de la leucine entre les différentes voies métaboliques est modifiée et, notamment, la quantité de leucine perdue par oxydation est plus importante. II serait intéressant de déterminer les facteurs susceptibles de réduire ces pertes, de façon à augmenter l'efficacité alimentaire.

6e Réunion du groupe Développement I.N.R.A., Clermont-Ferrand/Theix, 22-23 mai 1980.

Remerciements. - Nous remercions. Y. Bonnel pour les analyses de gaz carbonique, J. Prugnaud pour les analyses radiocromatographiques des acides aminés, E. Aurousseau et F. Glomot pour leur aide technique.

\section{Références}

ALBERTSE E. C., GARLICK P. J., PAIN V. M., 1979. Whole-body protein synthesis and oxidation rates in steptozotocin diabetic rats. Proc. Nutr. Soc., 38, $125 \mathrm{~A}$.

ALRIC M., 1979. Etude des variations des différents flux de leucine en fonction de l'âge chez le rat : mise au point expérimentale. Rapp. Stage Fin Etudes, INSA Lyon.

EDMUNDS B. K., BUTTERY P. J., 1978. Protein turnover and whole-body nitrogen metabolism in the growing pig. Proc. Nufr. Soc., 37, 32A.

GARLICK P. J., BURK T. L., SWICK R. W., 1976. Protein synthesis and RNA in tissues of the pig. Am. J. Phys., 230, 1108-1112.

GARLICK P. J., CLUGSTON G. A., SWICK R. W., MEINERTZHAGEN I. H., WATERLOW J. C., 1978. Diurnal variations in protein metabolism in man. Proc. Nutr. Soc., 37, 33A.

GARLICK P. J., MILLWARD D. J., JAMES W. P. T., 1973. The diurnal response of muscle and liver protein synthesis in vivo in meal-fed rats. Biochem. J., 136, 935-945.

GOLDEN M. H. N., WATERLOW J. C., 1977. Total protein synthesis in elderly people : a comparison of results with ${ }^{15} \mathrm{~N}$ glycine and ${ }^{14} \mathrm{C}$ leucine. Clin. Sci., 53, 277-288.

GRIZARD J., PRUGNAUD J., ARNAL M., PION R., 1975. Effet de l'insuline sur la composition corporelle et les teneurs en acides aminés libres du sang, du foie et du muscle du rat en croissance soumis à une restriction énergétique. Ann. Biol. onim. Bioch. Biophys., 15, 569-582.

JAMES N. P. T., GARLICK P. J., SENDER P. M., WATERLOW J. C., 1976. Studies of amino acid and profein metabolism in normal man with L-(U-14 C) tyrosine. Clin. Sci. mol. Med., 50, 525-532.

JARRIGE J. F., BOUCHER D., LEINOT M., 1978. Cathétérismes vasculaires chroniques chez le rat. Adaptation aux injections et prélèvements sanguins semi-continus pour études hormonales. C. R. Soc. Chim. biol., 172, 919-926.

LO C. C., MILLWARD D. J., 1977. The effect of fasting and refeeding on whole-body protein synthesis in the rat. Proc. Nutr. Soc., 36, 137 A.

MC NURLAN M. A., GARLICK P. J., 1980. Contribution of rat liver and gastrointestinal tract to wholebody protein synthesis in the rat. Biochem. J., 180, 381-383.

NEALE R. J., WATERLOW J. C., 1973. Estimation of maintenance requirements of amino acids in the rat by measurement of the rate of ${ }^{14} \mathrm{C}$ oxidation in vivo. Fed. Proc., 32, $5 \mathrm{~A}$.

NICHOLAS G. A., LOBLEY G. E., HARRIS C. I., 1977. Use of the constant infusion technique for measuring rates of protein synthesis in the New Zealand white rabbit. Br. J. Nutr., 38, 1-17. 
OBLED C., ARNAL M., FAUCONNEAU G., 1975. Synthèse protéique in vivo dans divers tissus du rat en croissance en fonction du rythme alimentaire. Ann. Biol. anim. Bioch. Biophys., 15, 73-93.

O'KEEFE S. J. D., SENDER P. M., JAMES W. P. T., 1974. "Catabolic » loss of body nitrogen in response to surgery. The Lancet, 2, 1035-1037.

REEDS R. J., FULLER M. F., LOBLEY G. E., CADENHEAD A., Me DONALD J. D., 1978. Protein, synthesis and amino acid oxidation in growing pigs. Proc. Nutr. Soc., 37, 106A.

REEDS R. J., LOBLEY G. E., 1980 . Protein synthesis : are there real species differences ? Proc. Nutr. Soc. 39, 43-52.

RÉRAT A., FÉVRIER C., HENRY Y., LOUGNON J., 1964. Evolution de la composition corporelle chez le rat blanc en croissance. Ann. Biol. anim. Bioch. Biophys., 4, 35-47.

SMITH P. H., 1980. Comparative amino acid requirements. Proc. Nutr. Soc., 39, 71-78.

SNEDECOR G. W., COCHRAN W. G., 1971. Méthodes statistiques. A.C.T.A. éd. Paris.

WATERLOW J. C., STEPHEN J. M. L., 1967. The measurement of total lysine turnover in the rat by intravenous infusion of $L(U-14 C)$ lysine. Clin. Sci., 33, 489-506.

WATERLOW J. C., GARLICK P. J., MILLWARD D. J., 1978. Profein turnover in mammalian tissues and in the whole-body. North-Holland Publ. Co., Amsterdam-New York-Oxford.

WEEKS J. R., 1972. Methods in psychobiology, 2, Acad. Press. 